\title{
STUDY OF CLINICAL, LABORATORY PROFILE AND OUTCOME OF DENGUE FEVER ADMITTED IN PAEDIATRIC WARD OF A TERTIARY CARE HOSPITAL OF WESTERN UTTAR PRADESH, INDIA
}

\author{
Mohammad Kashif', Shaad Abqari², Zeeba Zaka-Ur-Rab33, Sadaf Mirza ${ }^{4}$ \\ ${ }^{1}$ Assistant Professor, Department of Paediatrics, Mayo Institute of Medical Sciences, Gadia, Barabanki. \\ ${ }^{2}$ Assistant Professor, Department of Paediatrics, JNMCH, AMU, Aligarh. \\ ${ }_{3}^{3}$ Associate Professor, Department of Paediatrics, JNMCH, AMU, Aligarh. \\ ${ }^{4}$ Assistant Professor, Department of Pathology, Mayo Institute of Medical Sciences, Gadia, Barabanki.
}

ABSTRACT

\section{BACKGROUND}

Dengue Fever epidemic resurfaced this year after 2005 with more number of cases and with varying presentations and complications. The presentation can vary from a mild febrile illness to severe refractory shock or severe bleeding. Dengue is an important cause of mortality and morbidity in India. Many recent studies have shown varied clinical manifestations from different geographical locations. The present prospective observational study was conducted to find out the clinical features, complications, response to treatment and outcome of patients suffering from dengue in a tertiary care centre of Western Uttar Pradesh, India. Objective-To study the clinical, pathological, laboratory profile and outcome of the confirmed cases of Dengue fever in Paediatric age group admitted in the hospital.

\section{MATERIALS AND METHODS}

The Prospective observational study was performed at Department of Paediatrics, Jawaharlal Nehru Medical College, Aligarh Muslim University, Aligarh which is a tertiary care hospital of Western Uttar Pradesh. The study period was from June 2013 to November 2013. Patients up to 18 years of age who were admitted in Paediatric ward and were positive for serological test (NS1 Ag, IgM /IgG) for dengue fever were included. The patients were subjected to detailed history and examination and relevant investigations were sent.

\section{RESULTS}

Total 126 patients were included. Out of these, 66 were male (52.38\%) and 20 were female (47.61\%). Majority of them presented in critical phase on day 4-6 of illness (57.14\%). Most of the cases were admitted in post monsoon period in the month of October and November. The most common presentation was fever $126(100 \%)$ followed by petechial rash 75 (59.52\%). Pruritus was present in $60(47.61 \%)$ patients. NS1 antigen was positive in majority of patients $(78,61.9 \%)$. Thrombocytopenia was the commonest haematological abnormality. Deranged hepatic, renal and cerebral functions were noted. Total leucocyte counts were less than 4000 in most of the patients $(54,42.8 \%)$. Bleeding manifestations in the form of gum bleeding, haematuria, epistaxis, melaena were present mainly in patients having severe thrombocytopenia $(<50,000 / \mathrm{cu} \mathrm{mm})$. On ECG $39(23.8 \%)$ patients were having myocarditis whereas 24 (19\%) patients had pleural effusion on chest x-ray. The average duration of stay in the hospital was 6-7 days and majority of the patients were discharged on day 9-10 of illness. Comorbid conditions in the form of malaria 9 (7.1\%) and enteric fever 7 (5.5\%) were also noted. Blood cultures were positive in only 8 (6.3\%). 8 patients expired and mortality rate was 6.3\%. Shock, bleeding, encephalitis, ARDS, late presentation and diagnosis were associated with poor outcome and mortality.

\section{CONCLUSION}

Dengue is an important cause of mortality and morbidity in western Uttar Pradesh and adjoining areas. Community awareness, early diagnosis and management and vector control measures need to be strengthened, during peri-monsoon period, in order to curb the increasing number of dengue cases. High index of suspicion, monitoring of the clinical and laboratory parameters and prompt intervention may help in reducing the mortality.

\section{KEYWORDS}

Dengue Fever, Platelets, Clinical and Laboratory Parameters, Dengue Complications, DHF, DSS.

HOW TO CITE THIS ARTICLE: Kashif M, Abqari S, Rab ZZU, et al. Study of clinical, laboratory profile and outcome of dengue fever admitted in paediatric ward of a tertiary care hospital of western Uttar Pradesh, India. J. Evolution Med. Dent. Sci. 2017;6(95):69826986, DOI: $10.14260 /$ jemds/2017/1515

\section{BACKGROUND}

"Dengue" word is derived from Swahili phrase ka-dinga pepo means "cramp like seizure". Due to symptoms of myalgia and

'Financial or Other Competing Interest': None.

Submission 09-11-2017, Peer Review 06-12-2017,

Acceptance 11-12-2017, Published 23-12-2017.

Corresponding Author:

Sadaf Mirza,

Department of Pathology,

Mayo Institute of Medical Sciences, Gadia, Barabanki.

E-mail: sadafazmi2@gmail.com

DOI: $10.14260 / j e m d s / 2017 / 1515$

Arthralgia, it was first reported by Benjamin Rush in Philadelphia as "Back bone fever".[1] Dengue fever is an acute viral illness, mostly prevailing in tropical and subtropical countries. It is currently the second most prevalent vector borne disease in the world[2] posing threat to nearly half of world population. Dengue has recently become a major public health problem causing significant morbidity, mortality and economic loss. Worldwide around 2.5 billion people live in dengue prone regions and about 100 million new cases are detected each year with 500000 cases of DHF and an estimated 22000 dengue-related deaths. [3] Dengue is endemic in more than 100 countries. Recent demographic, geographic changes 
and increased urbanisation have contributed to the increased occurrence of Dengue fever. ${ }^{[4]}$ Dengue is prevalent in India since last two centuries but first evidence of occurrence is from Vellore district in Tamil Nadu during 1956.[5].Since last 10 years there has been increasing occurrence of dengue cases. In last decade, major outbreaks and deaths have occurred in Northern India (Haryana, Punjab, New Delhi, Uttar Pradesh), Southern India (Andhra Pradesh, Tamil Nadu and Karnataka), Western India (Gujarat, Rajasthan) and Eastern India (West Bengal).

Dengue is a vector-borne viral disease affecting humans through mosquito bite. There are four serotypes of dengue virus, DEN-1, DEN-2, DEN-3, DEN-4 belonging to the genus Flavivirus transmitted by Aedes aegypti responsible for Dengue fever (DF) and its more severe form i.e. dengue haemorrhagic fever (DHF). Incubation period of Dengue fever ranges from 3 to 15 (usually 5-8) days and transmission occurs mostly in monsoon and post monsoon season.[6] Infection by one serotype generates lifelong immunity against the same serotype, but gives transient/partial protection against the other serotypes. ${ }^{[7,8]}$ Sequential infection with another serotype can result in the more severe DHF.[9] Clinical manifestation is varied and range from self-limiting flu-like illness called Dengue fever to severe often with unpredictable symptoms in DHF/DSS.[10]. Typical dengue fever is characterised by high fever, severe headache, myalgia, arthralgia, maculopapular rash and retro-orbital pain. About $5-10 \%$ patients progress to DHF which is characterised by high-grade fever, haemorrhagic phenomenon (presence of at least one i.e. positive tourniquet test; petechiae, ecchymoses, or purpura; epistaxis or bleeding from mucosa, gastrointestinal tract, injection sites or others), thrombocytopenia (moderate to marked drop in platelets), and haemoconcentration (raised Packed Cell Volume). Plasma leakage may result in pleural effusion and ascites. DHF is characterised by onset of dramatic haemorrhagic manifestations. DSS is most severe form of DHF that is due to significant intravascular volume depletion, haemodynamic compromise, poor organ and tissue perfusion.[11] Severe dengue can also produce hepatic damage, encephalopathy, encephalitis and cardiomyopathy, although these manifestations are rare, but the risk of death in such cases is high. Both DHF/DSS can cause high mortality if not managed urgently and appropriately.[12,13] There are no specific antiviral treatments for dengue infection and patients usually recover with timely fluid and electrolyte replacement.[14] Hence, clinicians must be able to identify the warning signs of dengue fever like severe abdominal pain, tenderness, persistent vomiting, mucosal bleeding, liver enlargement $>2$ $\mathrm{cm}$, clinical fluid accumulation, lethargy, restlessness, increase in haematocrit with rapid decrease in platelet counts, for the better management of dengue cases.[15] Timely and appropriate case management can reduce the mortality to below 1\%.[14] Paucity of data in paediatric age groups and varied clinical presentations and early complications lead to increased case fatality i.e. above $1 \%$ in paediatric age group over last 10 years.[16] The resurgence of dengue has been observed in North India and varied clinical presentations are being reported in the outbreaks reported from different geographical locations.[17-23] The present study was conducted to evaluate the clinical, laboratory profile and outcome of dengue fever in paediatrics age group in western Uttar Pradesh region of India.

\section{MATERIALS AND METHODS}

This Prospective observational study was performed at Department of Paediatrics, Jawaharlal Nehru Medical College, Aligarh Muslim University, Aligarh which is a tertiary care hospital of western Uttar Pradesh. The centre receives patients from western Uttar Pradesh, which is very close to New Delhi and NCR region. The study period was of approximately 6 months from June 2013 to November 2013 in which dengue fever was more prevalent. Patients of paediatrics age group 0 to 18 years who were antigen positive (NS1 Ag) or antibody positive (IgM \& IgG) were included in the study. All such patients who were admitted in the Paediatric ward of hospital underwent detailed clinical examination and investigation. Patients were assessed for clinical manifestations such as fever, along with other cardinal symptoms like headache, pain abdomen, skin bleeds, rash over body, anorexia, nausea/vomiting, myalgia, joint pain and retro-orbital pain. The patients were subjected to routine laboratory tests such as complete haemogram, liver function test, renal function test, serum electrolytes, blood cultures and urine microscopy test. Serial platelet count and haematocrit levels were monitored during the hospital stay. The patients were also subjected to radiological and other investigations when clinically warranted. Complications at any stage of dengue fever like shock, bleeding, pleural effusion, encephalopathy, myocarditis were recorded. Other relevant information like patient's demography, treatment given including platelets transfused and the ultimate outcome i.e. death or discharge were also recorded. The patients were also investigated for other common causes of fever endemic in our region such as Malaria, Typhoid and Leptospirosis. The collected data were analysed by using Microsoft Excel and SPSS 17.

\section{RESULTS}

Of all the total 126 patients admitted during the study period, 66 were male $(52.38 \%)$ and 60 were female $(47.61 \%)$ (Table 1). Majority of them presented on day 4-6 of illness (57.14\%) which is the critical phase of dengue fever. Most of the patients admitted were of age group 8 to 12 yrs. (Table 1). Majority of the cases were admitted in October and November which is the post monsoon period. (Table 2).

The most common presentation was fever 126 (100\%) followed by petechial rash 75 (59.52\%). Vomiting was present in 72 patients $(57.14 \%)$, headache and myalgia were noted in 51 patients $(40.47 \%)$. Abdominal pain was complained by 48 patients $(38.9 \%)$. Pruritus was present in $60(47.61 \%)$ cases. 39 (30.9\%) patients were having features of myocarditis on ECG and out of these patients, shock was reported in 21 (16.66\%) patients. In bleeding manifestations, melaena was noted in $21(16.66 \%)$ patients, haematuria in $12(9.5 \%)$ patients, gum bleeding was reported in $8(6.3 \%)$ cases whereas epistaxis was present in $15(11.9 \%)$ patients. 44 (34.9\%) patients were having features of hepatitis, in the form of raised SGOT( $>40 \mathrm{U} / \mathrm{L}$ ) and raised SGPT( $>40 \mathrm{U} / \mathrm{L})$. In central nervous system manifestations, seizures were reported in 9 (7.14\%) patients whereas frank features of encephalopathy were present in $6(4.7 \%)$ patients. 13 patients $(10.3 \%)$ were having features of renal failure. 20 patients $(15.87 \%)$ were also having breathing difficulty in the form of tachypnoea and on chest x-ray $24(19.1 \%)$ patients were having pleural effusion. 
Only NS1 antigen positivity was present in 78 patients (61.9\%), as most of the patients presented early. Antigen NS1 and IgM were positive in 15 (11.9\%), NS1, IgM and IgG were positive in $18(14.2 \%)$ patients. Most common haematological manifestation was thrombocytopenia $(<1.5$ lakhs/cu. mm) which was present in 111 (88.0\%) patients. Severe thrombocytopenia $(<50,000$ thousand/cu. $\mathrm{mm}$ ) was noted in 54 (42.8\%) patients. Most of the patients were having bleeding manifestations, $56(44.4 \%)$ in the form of melaena, gum bleeding, epistaxis and haematuria with severe thrombocytopenia. Mean or average platelet count was 75000 /cu. mm on day 4 of illness and was lowest on day 6 of illness at $51000 / \mathrm{cu}$. mm. Platelet count were on increasing trend from day 7 of illness. Haemoglobin was more than $12 \mathrm{mg} / \mathrm{dL}$ in $105(83.3 \%)$ and only $12(9.5 \%)$ patients were having haemoglobin less than $10 \mathrm{mg} / \mathrm{dL}$. Haematocrit more than 40 was observed in 39 (30.9\%) patients. Total leucocyte counts were less than 4000 in $54(42.8 \%)$ patients and more than 4000 in $72(57.1 \%)$ patients. Only $12(9.5 \%)$ patients were having leucocytosis (>11000/cu. mm). Deranged liver functions were observed i.e. raised serum bilirubin $(>1.0$ $\mathrm{mg} / \mathrm{dL}$ ) in 10 (7.9\%) patients. Renal functions were deranged as serum creatinine $(>1.5 \mathrm{mg} / \mathrm{dL})$ was noted in $13(10.3 \%)$ patients.

On ECG, 39 (23.8\%) patients were having features suggestive of myocarditis whereas 24 (19\%) patients on chest $\mathrm{x}$-ray shows pleural effusion. The average duration of stay in hospital was 6-7 days and majority of patients were discharged on day 9-10 of illness. Malaria (QBC) was positive in only 9 cases and 7 patients were associated with enteric fever and were Widal positive. Blood culture was positive in only 8 patients. 8 patients expired and mortality rate was $6.3 \%$. Patients who expired were actually those patients, who presented late and had complications like shock, ARDS, encephalopathy and bleeding.

\begin{tabular}{|c|c|c|c|}
\hline Age Group & Male & Female & Total \\
\hline $0-4$ & $8(6.3 \%)$ & $10(7.9 \%)$ & $18(14.2 \%)$ \\
\hline $4-8$ & $24(19.1 \%)$ & $16(12.6 \%)$ & $40(31.7 \%)$ \\
\hline $8-12$ & $34(26.9 \%)$ & $34(26.9 \%)$ & $68(53.9 \%)$ \\
\hline Total & $\mathbf{6 6}$ & $\mathbf{6 0}$ & $\mathbf{1 2 6}$ \\
\hline \multicolumn{4}{|c|}{ Table 1. Age and Sex wise Distribution of Dengue Cases } \\
\hline
\end{tabular}

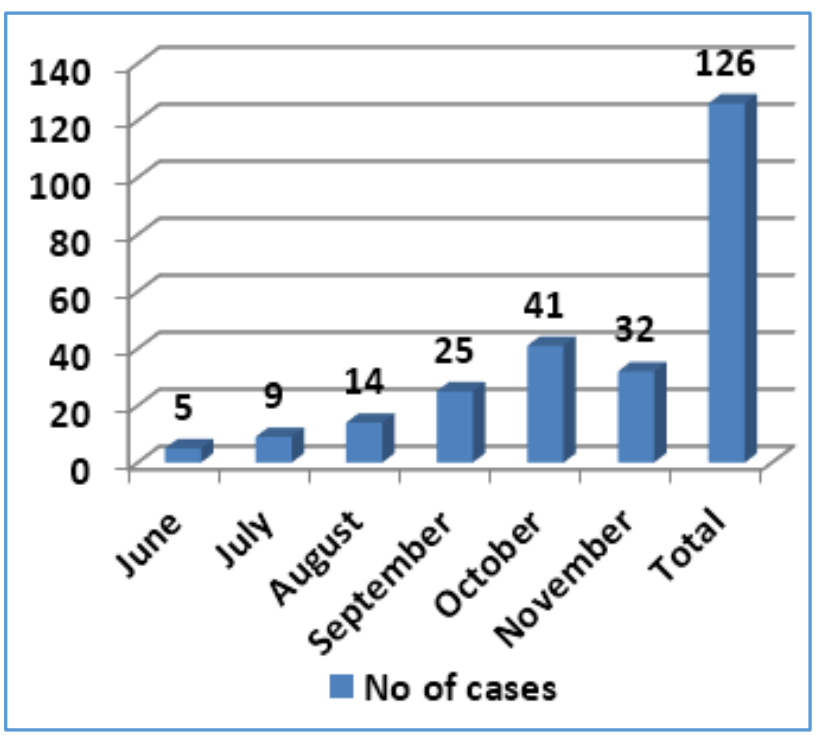

Graph 1. Monthly Distribution of Dengue Cases

\begin{tabular}{|c|c|}
\hline Month & No of Cases (\%) \\
\hline June & $5(3.96 \%)$ \\
\hline July & $9(7.14 \%)$ \\
\hline August & $14(11.1 \%)$ \\
\hline September & $25(19.8 \%)$ \\
\hline October & $41(32.5 \%)$ \\
\hline November & $32(25.3 \%)$ \\
\hline Total & $\mathbf{1 2 6}(\mathbf{1 0 0 \% )}$ \\
\hline Table 2. Month wise Distribution of Dengue Cases \\
\hline
\end{tabular}

\begin{tabular}{|c|c|}
\hline Clinical Manifestations & No. of Cases (\%) \\
\hline Fever & $126(100 \%)$ \\
\hline Petechial rash & $75(59.5 \%)$ \\
\hline Nausea and vomiting & $72(57.1 \%)$ \\
\hline Headache & $51(40.4 \%)$ \\
\hline Myalgia and fatigue & $51(40.4 \%)$ \\
\hline Abdominal pain & $48(38 \%)$ \\
\hline Pruritus & $60(47.6 \%)$ \\
\hline Retro orbital pain & $35(27.7 \%)$ \\
\hline Joint pain & $49(38.8 \%)$ \\
\hline Epistaxis & $15(11.9 \%)$ \\
\hline Melaena & $21(16.6 \%)$ \\
\hline Haematuria & $12(9.5 \%)$ \\
\hline Gum bleeding & $8(6.3 \%)$ \\
\hline Shock & $21(16.6 \%)$ \\
\hline Myocarditis (on ECG) & $39(30.9 \%)$ \\
\hline Pleural effusion on X-ray & $24(19.1 \%)$ \\
\hline Hepatitis & $44(34.9 \%)$ \\
\hline Seizure & $9(7.1 \%)$ \\
\hline Encephalopathy & $6(4.7 \%)$ \\
\hline Renal failure & $13(10.3 \%)$ \\
\hline Table 3. Distribution of Clinical Manifestations and \\
Complications of Dengue Fever \\
\hline
\end{tabular}

\begin{tabular}{|c|c|}
\hline Laboratory Parameters & No. of Cases (\%) \\
\hline Haemoglobin $>12 \mathrm{mg} / \mathrm{dL}$ & $105(83.3 \%)$ \\
\hline Haemoglobin $<10 \mathrm{mg} / \mathrm{dL}$ & $12(9.5 \%)$ \\
\hline Haematocrit $>40$ & $39(30.9 \%)$ \\
\hline Platelet count $<1.5$ lakhs/cu.mm & $27(21.4 \%)$ \\
\hline Platelet count $<1.0$ lakhs/cu. $\mathrm{mm}$ & $30(23.8 \%)$ \\
\hline Platelet count $<50$ thousands /cu. mm & $54(42.8 \%)$ \\
\hline Platelet count $>1.5$ lakhs/cu.mm & $15(11.9 \%)$ \\
\hline Leucopenia $<4000 / \mathrm{cu} . \mathrm{Mm}$ & $54(42.8 \%)$ \\
\hline Leucocytosis $>11000 /$ cu.mm & $12(9.5 \%)$ \\
\hline $\mathrm{SGOT}>40 \mathrm{U} / \mathrm{L}$ & $44(34.9 \%)$ \\
\hline SGPT $>40 \mathrm{U} / \mathrm{L}$ & $38(30.1 \%)$ \\
\hline Serum bilirubin $>1.0 \mathrm{mg} / \mathrm{dL}$ & $10(7.9 \%)$ \\
\hline \begin{tabular}{|l|} 
Deranged RFT (Serum creatinine $>1.5$ ) \\
\end{tabular} & $13(10.3 \%)$ \\
\hline Widal positive & $7(5.5 \%)$ \\
\hline Malaria (QBC) positive & $9(7.1 \%)$ \\
\hline Blood culture positive & $8(6.3 \%)$ \\
\hline Mortality & $8(6.3 \%)$ \\
\hline \multicolumn{2}{|c|}{$\begin{array}{c}\text { Table 4. Laboratory Parameters Including Haematological } \\
\text { and Biochemical Findings and Outcome in Dengue Cases }\end{array}$} \\
\hline Dengue Antigen and Antibody Positivi & \begin{tabular}{l|l|} 
ty & No. of cases (\%) \\
\end{tabular} \\
\hline NS1 Ag only & $78(61.9 \%)$ \\
\hline IgM & $3(2.3 \%)$ \\
\hline IgG & $1(0.8 \%)$ \\
\hline NS1 Ag, IgM, IgG & $18(14.2 \%)$ \\
\hline IgM and IgG only & $8(6.3 \%)$ \\
\hline NS1 Ag and IgM & $15(11.9 \%)$ \\
\hline \multicolumn{2}{|c|}{$\begin{array}{c}\text { Table 5. Pattern of NS1 Ag, IgM, IgG Positivity of Dengue } \\
\text { Cases }\end{array}$} \\
\hline
\end{tabular}




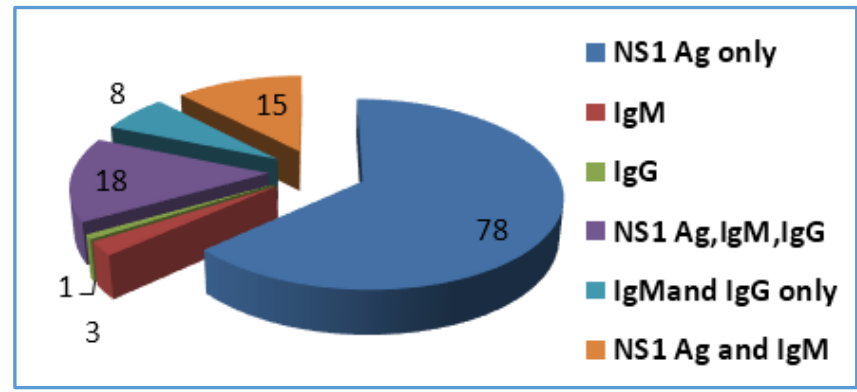

Graph 2. Pattern of NS1 Ag, IgM, IgG Positivity of Dengue Cases

\section{DISCUSSION}

Dengue fever is an emerging vector borne disease in tropical and subtropical regions. Its manifestation varies from a selflimiting viral fever to more critical complications like dengue haemorrhagic fever (DHF), dengue shock syndrome (DSS). Dengue virus is transmitted by its vector mosquito named Aedes aegypti. Major outbreaks occurred in monsoon and post monsoon period which are favourable conditions for its vector. Due to urbanisation and changes in habitat there is enough water logging and favourable factors for growth of mosquitoes. In the present study, maximum number of cases are reported in the September to November period which is a favourable condition for growth of vector Aedes aegypti. Monsoon and post monsoon period is favourable for transmission when number of cases increase. This fact is clearly evident in the present study and the previous studies conducted.[24] Similar results were shown in another study done in Kolkata.

Several studies conducted showed male preponderance among affected individuals as seen in the study conducted by Ashwini $\mathrm{K}$ et al i.e. 1.8:1.[24] Anugha G Kinikar's study shows $1.3: 1^{[25]}$ but in our study male to female ratio is $1.1: 1$. This difference in ratio may be due to the paediatric age group where other factors like outdoor working, activity and exposure could not be applied. NS1 antigen reactive patients were more in number as compared to IgM and IgG antibody positive patients as most of the patients presented on day 4-5 of illness. Similar results were shown in the study conducted by Anugha G Kinikar et al[25] and K M Mohamed et al.[26]

In the present study, fever was the most common presenting symptom as it was present in 126 patients (100\%). This finding was consistent with other studies conducted by Singh $\mathrm{R}$ et al, Ashwini $\mathrm{K}$ et al[24] and KM Mohamed et al.[26] Abdominal symptoms such as abdominal pain was present in $48(38 \%)$, nausea and vomiting in $72(57.1 \%)$ patients. Similar results were shown in studies done by Ashwini $\mathrm{K}$ et al[24] and KM Mohamed et al.[26] In our study, other symptoms like headache, myalgia and fatigue were present in around $40.4 \%$ patients, these findings were similar to studies done by KM Mohamed et al[26] and Neerja $M$ et al.[27] However, Singh R and KM Mohamed showed slightly higher no. of patients with headache, myalgia and headache respectively i.e. around $76 \%$.

Retro-orbital pain was present in $27.7 \%$ patients but KM Mohamed et al[26] and Denys Eiti Fugimoto reported slightly lower incidence i.e. $12.32 \%$ and $16.1 \%$ [28] respectively. Bleeding manifestations in the form of melaena, epistaxis, gum bleed, haematuria were present in total $44.4 \%$ cases as Ashwini Ket al[26] reported $26.6 \%$, but KM Mohamed et al and Tejeshree A et al reported $9.58 \%$ and $3.84 \%{ }^{[29]}$ respectively.
These differences may be due to this study sample being of paediatrics age group, it is correlating with severe thrombocytopenia $(<50,000 / \mathrm{cu}$. $\mathrm{mm})$ which is present in $42.8 \%$ cases.

Pleural effusion on x-ray was present in $19.15 \%$ patients. Similar finding was noted by KM Mohamed et al, $13.69 \%$. Renal failure was noted in $10.3 \%$ patients; however, KM Mohamed noted only $1.36 \%$ and Singh R et al[30] noted $7.1 \%$, but in other studies percentage of renal failure was as high as $40.6 \%$. CNS involvement of dengue causes seizure, encephalitis. In our study, seizure was noted in $7.1 \%$, encephalopathy in $4.7 \%$. Similar results (8.9\%) were reported by Singh R et al[30] and KM Mohamed et al. Hypotension and shock was noted in $16.6 \%$.

Laboratory investigations were evaluated in our study, most of the patients were having thrombocytopenia. It is present in as many as $88 \%$, whereas Rashmi KS et al[31] reported $72.77 \%$, but similar result was noted by KM Mohamed and Singh $\mathrm{R}$ et al. Increased haematocrit was observed in $30.9 \%$, these findings were similar to study done by Anuradha $\mathrm{M}$ et al (30\%).[32] Whereas higher numbers were observed by KM Mohamed et al (57.53\%). Anaemia was reported in $9.5 \%$ patients, these results were consistent with KM Mohamed et al, but Tejushree A et al reported higher incidence which shows significant difference in both studies. Leucopenia was observed in $42.8 \%$ of patients, similar to KM Mohamed et al (43.83\%) whereas Prafulla Dutta et al reported $30 \%$.[33] Liver dysfunction was noted in many patients, SGOT is slightly higher than SGPT. This finding is also obvious in our study as SGOT is deranged in $34.9 \%$ and SGPT in $30.1 \%$. Liver enzymes were deranged in higher number of patients whereas only $7.9 \%$ had deranged or elevated bilirubin level. These findings were similar to study done by Prafulla Dutta et al.[33]

Our patients were also investigated for other endemic causes of fever prevalent in our region like malaria and typhoid along with dengue which also causes thrombocytopenia and often lead to delay in diagnosis. Malaria was present in $7.1 \%$, typhoid in $5.5 \%$. Blood culture was only positive in $6.3 \%$ patients. Mortality rate was $6.3 \%$ in our study. Similar results were reported by Singh R et al, 3.6\%[30]. Another study from Kerala reported mortality rate of $3.2 \%$ whereas earlier studies showed higher mortality rate of $20 \%$.[34] Most of the patients who expired were presented late and were having complications like shock, encephalitis, bleeding and myocarditis. Our study shows similar results as compared to other studies in other regions. However, some presentations and laboratory parameters were different from other studies. This may be due to the reason that our study was conducted in paediatrics age group whereas others were conducted in older age groups.

\section{CONCLUSION}

Dengue is an important cause of mortality and morbidity in western Uttar Pradesh and adjoining areas. Community awareness, early diagnosis and management and vector control measures need to be strengthened, during perimonsoon period, in order to curb the increasing number of dengue cases. High index of suspicion, monitoring of the clinical and laboratory parameters and prompt intervention may help in reducing the mortality. 


\section{REFERENCES}

[1] Gupta N, Srivastava S, Jain A, et al. Dengue in India. Indian J Med Res 2012;136(3):373-90.

[2] Jelinek T. Dengue fever in International travelers. CID 2000;31:144-7.

[3] Kim K, Gina S, Miriam RE. Fever pitch: Mosquito-Borne dengue fever threat spreading in the Americas. NRDC 2009.

[4] Gunusekaran P, Kaveri K, Mohana S, et al. Dengue disease status in Chennai (2006-2008): a retrospective analysis. Indian J Med Res 2011;133(3):322-5.

[5] Sharma SK, Gautham A. Dengue fever in India: an overview. Medicine update 2010;20:657-9.

[6] Ghazala Z, Anuradha HV, Shivamurthy MC. Pattern of management and outcome of dengue fever in pediatric in-patients in a tertiary care hospital: a prospective observation study. Int J Basic Clin Pharmacol 2014;3(3):534-8.

[7] Halstead SB. Pathogenesis of dengue: challenges to molecular biology. Science 1988;239(4839):476-81.

[8] Kurane I. Dengue haemorrhagic fever with special emphasis on immunopathogenesis. Comp Immunol Microbiol Infect Dis 2007;30(5-6):329-40.

[9] WHO. Comprehensive guidelines for prevention and control of dengue and dengue haemorrhagic fever. $2^{\text {nd }}$ edn. Geneva: World Health Organization, 2011.

[10] Siddharth NS, Yash PM, Surendra KS, et al. API Textbook of Medicine. $7^{\text {th }}$ edn. New Delhi: Jaypee Brothers Medical Publishers (P) Ltd., 2003.

[11] Kurukularatne C, Dimatatac F, DLT Teo, et al. When less is more: can we abandon prophylactic platelet transfusion in Dengue fever? Ann Acad Med Singapore 2011;40:539-45.

[12] Gubler DJ. Dengue and dengue haemorrhagic fever. Clin Microbiol Rev 1998;11(3):480-96.

[13] Lum LC, Lam SK, Choy YS, et al. Dengue encephalitis: a true entity? Am J Trop Med Hyg 1996;54(3):256-9.

[14] Solomon T, Dung NM, Vaughn DW, et al. Neurological manifestations of dengue infection. Lancet 2000;355(9209):1053-9.

[15] http://www.cdc.gov/dengue/redources/dengueclinici anguide $508 \mathrm{pdf}$

[16] Dengue: Guidelines for diagnosis, treatment, prevention and control-New edition. A joint publication of the WHO and TDR, 2009.

[17] Rajadhyaksha A, Mehra S. Dengue fever evolving into systemic lupus erythematosus and lupus nephritis: a case report. Lupus 2012;21(9):999-1002.

[18] Kanungo S, Shukla D, Kim R. Branch retinal artery occlusion secondary to dengue fever. Indian J Ophthalmol 2008;56(1):73-4.

[19] Matlani M, Chakravarti A. Changing trends of dengue disease: a brief report from a tertiary care hospital in New Delhi. Braz J Infect Dis 2011;15(2):184-5.

[20] Karoli R, Fatima J, Siddiqi Z, et al. Clinical profile of dengue infection at a teaching hospital in North India. J Infect Dev Ctries 2012;6(7):551-4.

[21] Daniel R, Rajamohanan, Philip AZ. A study of clinical profile of dengue fever in Kollam, Kerala, India. Dengue Bulletin 2005;29:197-202.
[22] Ratageri VH, Shepur TA, Wari PK, et al. Clinical profile and outcome of dengue fever cases. Indian J Pediatr 2005;72(8):705-6.

[23] Bandyopadhyay B, Bhattacharyya I, Adhikary S, et al. A comprehensive study on the 2012 dengue fever outbreak in Kolkata, India. ISRN Virology 2013;2013: p. 5.

[24] Kumar A, Rao CR, Pandit V, et al. Clinical manifestations and trend of dengue cases admitted in a tertiary care hospital, Udupi district, Karnataka. Indian Journal of Community Medicine 2010;35(3):386-90.

[25] Saini S, Kanikar AG, Deorukhkar S, et al. Epidemiology and seropositivity of dengue cases in a rural tertiary care hospital of western Maharashtra, India. IJBAR 2013;4(7):473-7.

[26] Kauser MM, Kalavathi GP, Mehul Radadiya, et al. A study of clinical and laboratory profile of dengue fever in tertiary care hospital in central Karnataka, India. Global Journal of Medical Research: B Pharma, Drug Discovery, Toxicology and Medicine 2014;14(5): version 1.

[27] Neeraja M, Lakshmi V, Teja VD, et al. Serodiagnosis of dengue virus infection in patients presenting to a tertiary care hospital. Indian J Med Microbiol 2006;24(4):280-2.

[28] Fujimoto DE, Koifman S. Clinical and laboratory characteristic of patients with dengue hemorrhagic fever manifestations and their transfusion profile. Rev Bras Hematol Hemoter 2014;36(2):115-20.

[29] Tejashree A, Thejaswini HS, Madhuri K. A serological study of Dengue and Hanta virus in acute febrile patients in a tertiary care hospital. International Journal of Pharmaceutical Science Invention 2014;3(7): 22-5.

[30] Singh R, Singh SP, Ahmad NZ. A study of clinical and laboratory profile of dengue fever in a tertiary care centre of Uttarakhand, India. Int J Res Med Sci International Journal of Research in Medical Sciences 2014;2(1):160-3.

[31] Rashmi KS, Jagdeesh, Ravikumar KL, et al. Serological markers prevalence and trend of probable dengue infection at a tertiary care hospital in Bangalore. Journal of Evolution of Medical and Dental Sciences 2013;12(36):6968-76.

[32] Mavilla A, Rahul HD. Screening and manifestations of seropositive dengue fever patients in perambalur: a hospital based study. International Journal of Medical Science and Public Health 2014;3(6):745-8.

[33] Prafulla D, Siraj AK, Jani B, et al. Demographic and clinical features of patients with dengue in Northeastern region of India: a retrospective crosssectional study during 2009-2011. Journal of Virology and Microbiology Article ID 786298, 2012;2012:1-11.

[34] WHO. Dengue guidelines for diagnosis, treatment, prevention and control WHO, 2009. http://whqlibdoc.who.int/publications/2009/978924 1547871eng.pdf. 2012. 